\title{
The EUI flight instrument of Solar Orbiter: from optical alignment to end-to-end calibration
}

J.-P. Halain, E. Renotte, F. Auchère, D. Berghmans, F. Delmotte, et al.

J.-P. Halain, E. Renotte, F. Auchère, D. Berghmans, F. Delmotte, L. Harra, W. Schmutz, U. Schühle, R. Aznar Cuadrado, C. Dumesnil, M. Gyo, T. Kennedy, C. Verbeeck, J. Barbay, B. Giordanengo, S. Gissot, A. Gottwald, K. Heerlein, M.-L. Hellin, A. Hermans, V. Hervier, L. Jacques, C. Laubis, A. Mazzoli, S. Meining, R. Mercier, A. Philippon, S. Roose, L. Rossi, F. Scholze, P. Smith, L. Teriaca, X. Zhang, P. Rochus, "The EUI flight instrument of Solar Orbiter: from optical alignment to end-to-end calibration," Proc. SPIE 10699, Space Telescopes and Instrumentation 2018: Ultraviolet to Gamma Ray, 106990H (6 July 2018); doi: 10.1117/12.2309339

Event: SPIE Astronomical Telescopes + Instrumentation, 2018, Austin, Texas, United States 


\title{
The EUI flight instrument of Solar Orbiter - From Optical Alignment to End-to-End Calibration
}

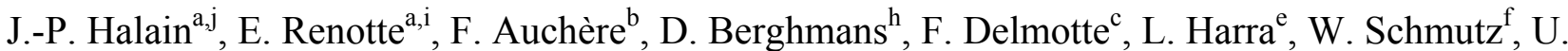

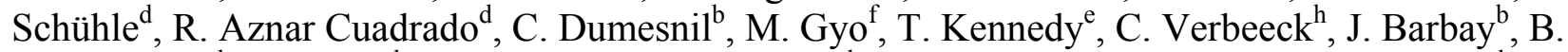 \\ Giordanengo $^{\mathrm{h}}$, S. Gissot ${ }^{\mathrm{h}}$, A. Gottwald ${ }^{\mathrm{g}}$, K. Heerlein ${ }^{\mathrm{d}}$, M.-L. Hellin ${ }^{\mathrm{a}}$, A. Hermans ${ }^{\mathrm{a}}$, V. Hervier ${ }^{\mathrm{b}}$, L.

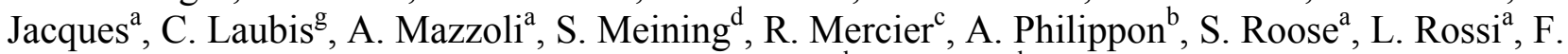

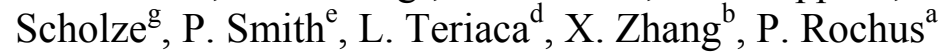 \\ ${ }^{a}$ Centre Spatial de Liège, Université de Liège, Angleur, Belgium \\ ${ }^{b}$ Institut d'Astrophysique Spatiale, Orsay, France \\ 'Laboratoire Charles Fabry, Institut d'Optique Graduate School, CNRS, Université Paris-Saclay, France \\ ${ }^{\mathrm{d}}$ Max-Planck-Institut für Sonnensystemforschung, Göttingen, Germany \\ ${ }^{e}$ UCL-Mullard Space Science Laboratory, Surrey, UK \\ ${ }^{f}$ Physikalisch-Meteorologisches Observatorium Davos and World Radiation Center, Switzerland \\ ${ }^{\mathrm{g}}$ Physikalisch-Technische Bundesanstalt, Berlin, Germany \\ ${ }^{h}$ Royal Observatory of Belgium, Brussels, Belgium \\ ${ }^{i} A M O S$ s.a., Liege Science Park, 4031 Angleur, Belgique \\ ${ }^{\mathrm{i}}$ European Space Agency, The Netherlands
}

\begin{abstract}
The Extreme Ultraviolet Imager (EUI) instrument for the Solar Orbiter mission will image the solar corona in the extreme ultraviolet $(17.1 \mathrm{~nm}$ and $30.4 \mathrm{~nm})$ and in the vacuum ultraviolet $(121.6 \mathrm{~nm})$ spectral ranges.

The development of the EUI instrument has been successfully completed with the optical alignment of its three channels' telescope, the thermal and mechanical environmental verification, the electrical and software validations, and an end-toend on-ground calibration of the two-units' flight instrument at the operating wavelengths.

The instrument has been delivered and installed on the Solar Orbiter spacecraft, which is now undergoing all preparatory activities before launch.
\end{abstract}

Keywords: Extreme Ultraviolet, Solar Orbiter, Flight Model, Optical Alignment, Calibration

\section{INTRODUCTION}

On-board the Solar Orbiter mission, the Extreme Ultraviolet Imager (EUI) ${ }^{[1][2][3][4][5][7][9][10][13]}$ is one of the six remotesensing instruments. The EUI instrument is dedicated to observations of the Solar Corona at the EUV wavelengths of $17.4 \mathrm{~nm}$ and $30.4 \mathrm{~nm}$, and the VUV wavelength of $121.6 \mathrm{~nm}$.

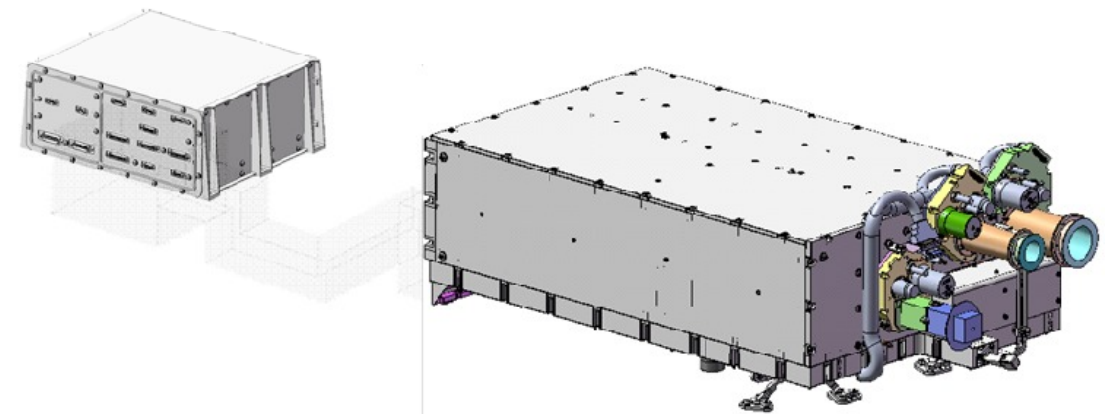

Figure 1 - Overview of the EUI instrument, composed of two units: the CEB (left) and the OBS (right).

Space Telescopes and Instrumentation 2018: Ultraviolet to Gamma Ray, edited by Jan-Willem A. den Herder, Shouleh Nikzad, Kazuhiro Nakazawa, Proc. of SPIE Vol. 10699, 106990H · @ 2018 SPIE CCC code: $0277-786 \mathrm{X} / 18 / \$ 18 \cdot$ doi: $10.1117 / 12.2309339$ 
The EUI instrument is composed of two units, a Common Electronic Box (CEB) and the Optical Bench System (OBS), as shown on Figure 1 and previously described ${ }^{[7]}$, these two units being electrically linked by a bundle of inter-connecting harness.

The OBS unit includes three channels, a Full Sun Imager (FSI) and two High Resolution Imagers (HRI). The optical design of these channels, all three of them with an off-axis mirror system, has been previously described ${ }^{[7][10]}$. Positioning and interferometric alignment of their respective mirrors were required during the assembly activities. Following the alignments of the three channels, the other sub-systems (cameras, mechanisms as shown in Figure 2) were integrated in the OBS unit.

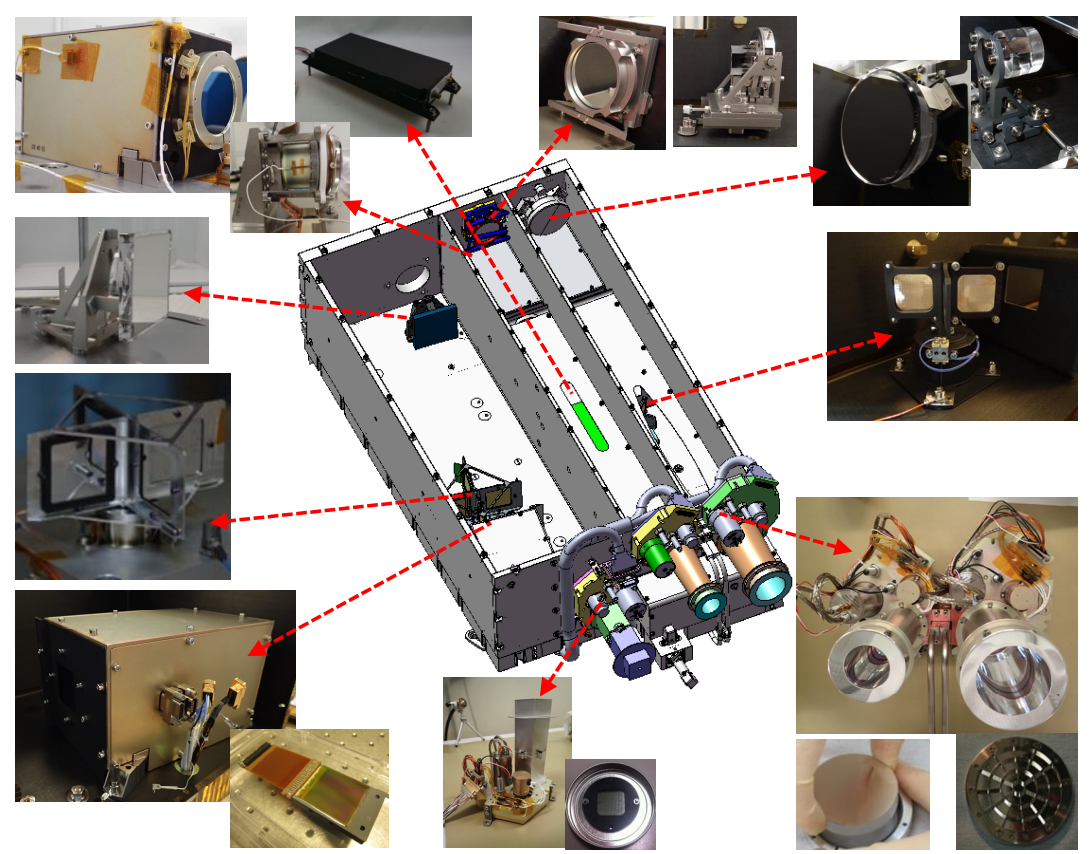

Figure 2 - Exploded view of the OBS unit showing its sub-systems.

The Assembly, Integration and Test (AIT) activities consisted of the classical acceptance tests: mechanical (vibrations), thermal (thermal cycling and bake-out) and electrical (EMC) environmental tests, and finally the end-to-end on-ground calibration at the operational wavelengths.

Following the completion of the AIT activities, the OBS (Figure 3) and CEB (Figure 4) Flight Model (FM) were then delivered to the Solar Orbiter platform at Airbus D\&S.

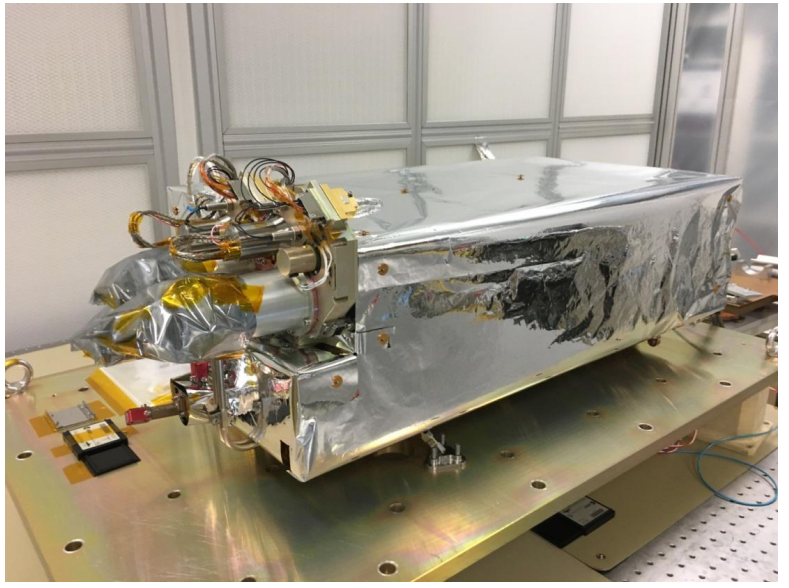

Figure 3 - The OBS FM unit.

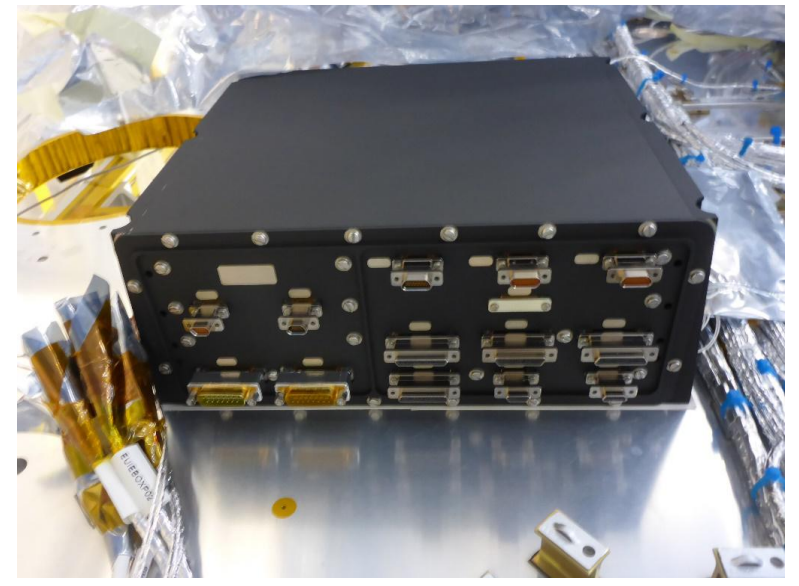

Figure 4 - The CEB FM unit. 


\section{OPTICAL ALIGNMENT ACTIVITIES}

The optical alignment of three channels mounted on a common structure is not a straightforward activity, in particular due to lack of easy access to the optical elements inside the housing. The alignment of the three channels has been achieved using different Optical Ground Support Equipment (OGSE): theodolites, interferometer and 3D measurement arm.

The Unit Alignment Reference Frame (UARF) and mechanical Unit Reference Frames (URF) of the EUI OBS unit are shown in Figure 5, with the three channels Line of Sight (LoS) and the Instrument Line of Sight (ILS). The horizontal $(\mathrm{Hz})$ and vertical $(\mathrm{V})$ tilt as used for the alignment are also shown (with yaw/pitch correspondence). In the following paragraphs the alignment of each channel is described.

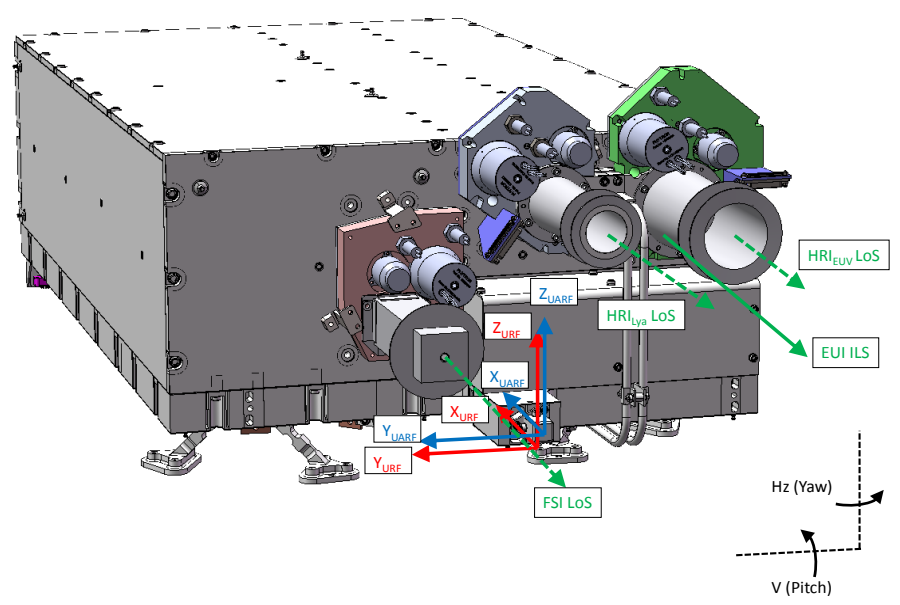

Figure 5 - EUI OBS unit reference frames.

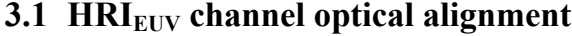

The two mirrors of the HRI Extreme UltraViolet (HRI $\mathrm{HUV}_{\mathrm{EU}}$ ) channel have first been positioned using theodolites to check their orientation w.r.t. the reference cube (which defines the UARF) and using a 3D arm to check their position w.r.t. the theoretical position predicted by the optical model. The mirror positions and orientations have been adjusted by shimming of the mirror mounts.

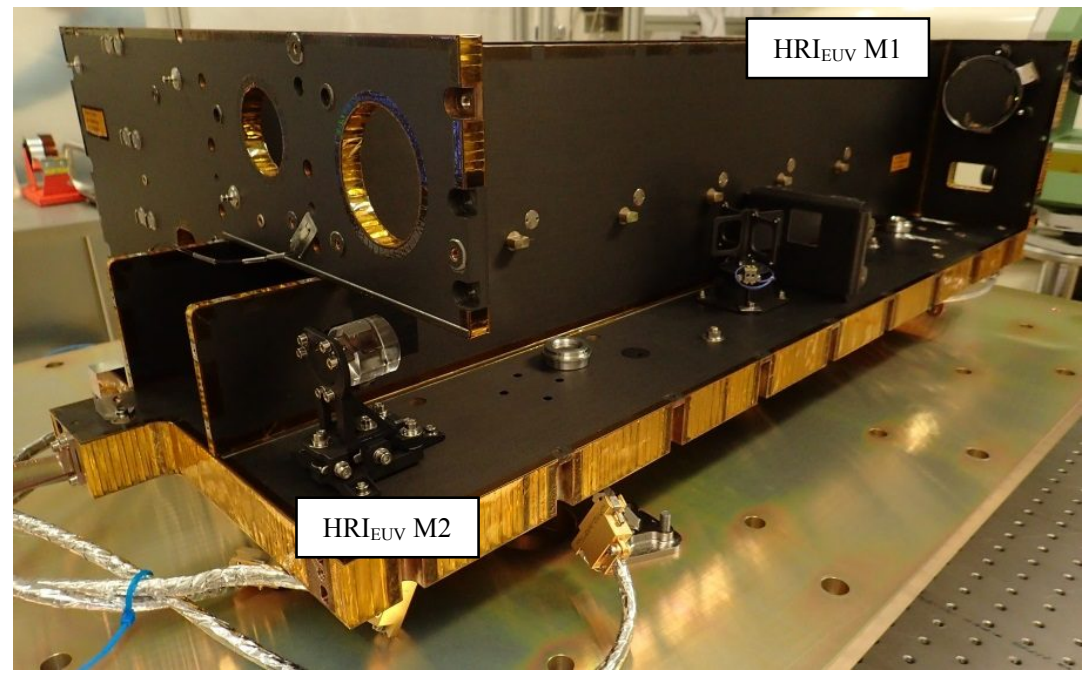

Figure 6 - EUI HRI $\mathrm{EUV}_{\mathrm{EV}}$ channel primary and secondary mirrors set in place inside OBS housing.

The foreseen 'front-side' interferometric alignment method ${ }^{[10]}$ uses a transmission flat on the interferometer to illuminate the instrument through its entrance aperture with a collimated beam. The obtained interferograms however showed unexpected parasitic patterns and aberration which made these measurements useless. 
It was thus decided to reverse the setup and illuminate the instrument with a spherical beam through its focal plane (Figure 7).

A first wave-front error (WFE) was measured to determine the residual focus term. From this residual term, the required displacement of the secondary mirror focus was calculated and applied with shims. The final WFE of the telescope was then measured (see Figure 8, the average result of $10 \mathrm{WFE}$ ). The Zernike coefficients of this averaged WFE are all below 0.01 waves, the Peak-Valley (PV) and Root Mean Square (RMS) amplitudes being within specifications with 0.057 waves $(\Leftrightarrow 36 \mathrm{~nm})$ and 0.009 waves $(\Leftrightarrow 5.7 \mathrm{~nm})$ respectively. For the final measurement, the flat orientation has been corrected by 16 arcsec in horizontal and 11 arcsec in vertical to cancel the tilt in the WFE. As a result, the measured WFE does not exactly correspond to the central part of the Field of View (FOV) of the instrument but it is very close to it (16 arcsec and 11 arcsec).

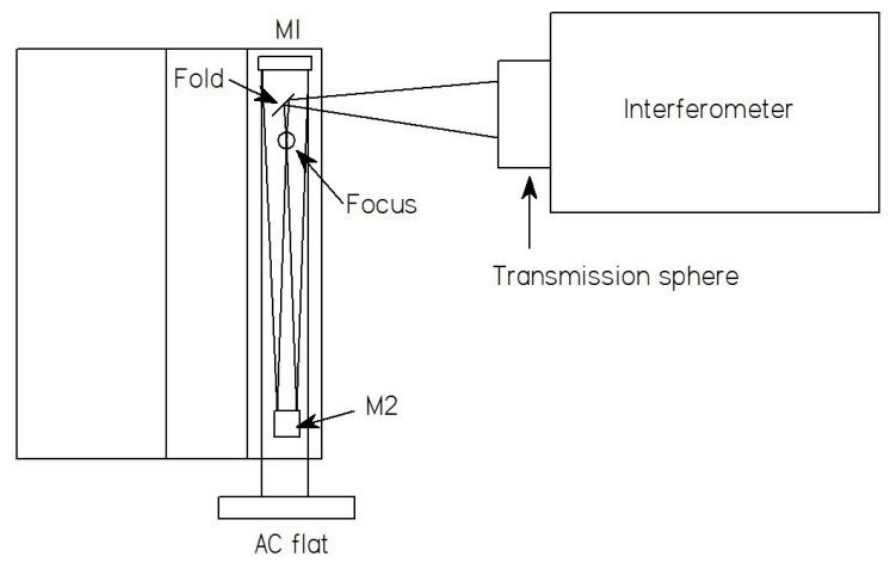

Figure 7 - $\mathrm{HRI}_{\mathrm{EUV}}$ interferometric back-side method.

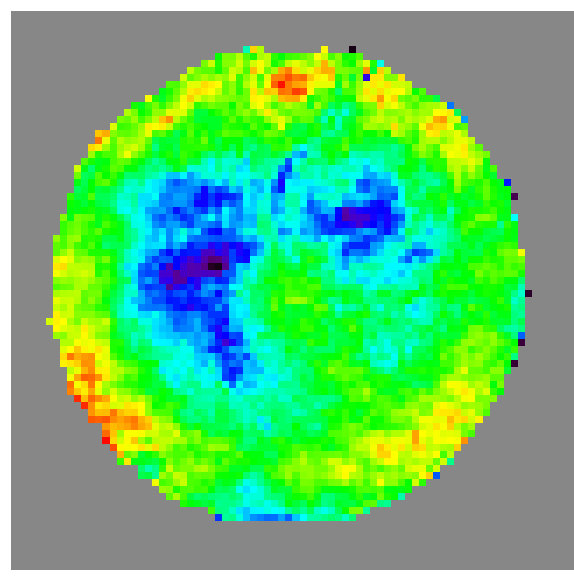

Figure 8 - Average of $\mathrm{HRI}_{\mathrm{EUV}}$ final alignment WFE.

The residual focus error of 0.009 waves corresponds to a defocus of $0.7 \mathrm{~mm}$ in the focal plane. The detector should thus be moved towards M2 for compensation. An additional $0.12 \mathrm{~mm}$ has also been included to be at the best focus for the whole FOV and not only the central part. A correction of $0.5 \mathrm{~mm}$ was implemented on the detector support structure, leaving $0.32 \mathrm{~mm}$ between the detector real position and the best focus position. From ray tracing simulations, this residual focus error gives an enlargement $<2 \mu \mathrm{m}$ on the image spot sizes (the RMS diameter is $7 \mu \mathrm{m}$ without residual focus), which is fully acceptable. Figure 9 shows the $\mathrm{HRI}_{\mathrm{EUV}}$ aligned channel with camera electronic around the detector.
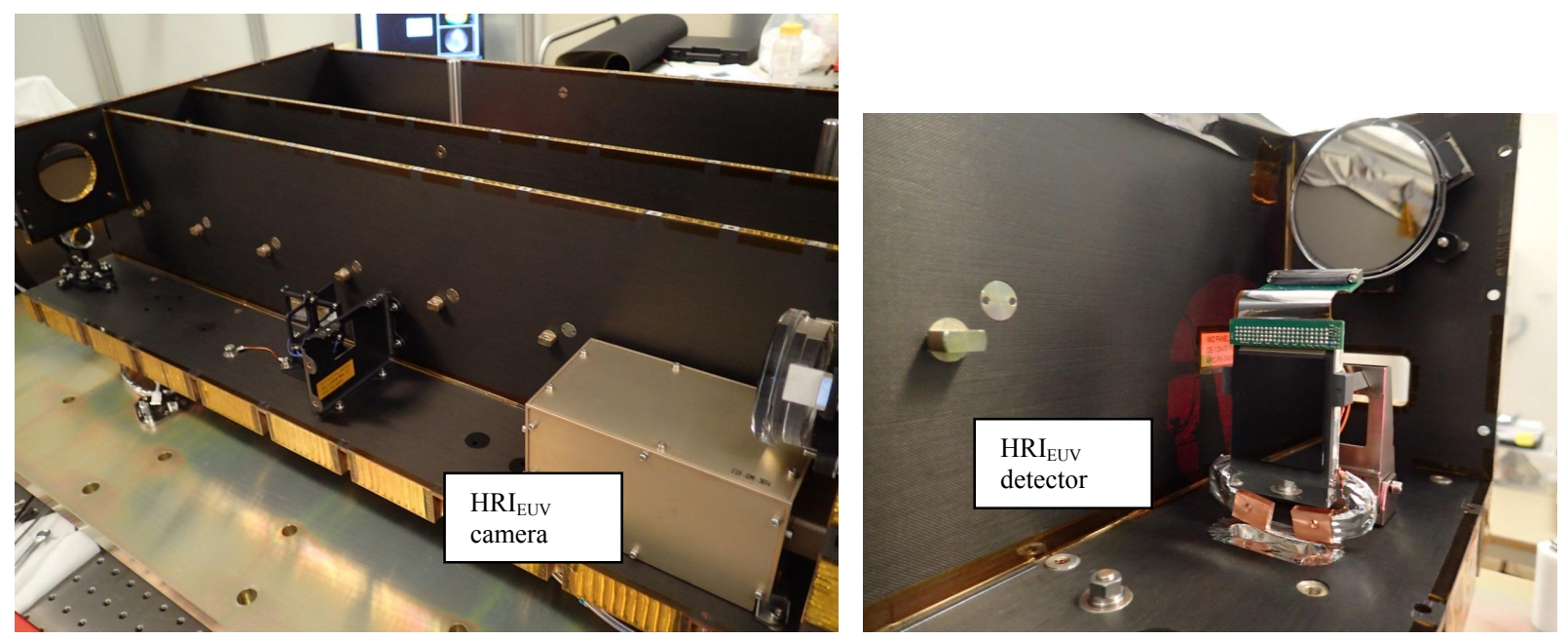

Figure 9 - Left: EUI HRI $\mathrm{EUV}_{\mathrm{V}}$ aligned channel with camera inside the OBS cavity. Right: Detector set in place at best focus. 


\subsection{HRI $_{\text {Lya }}$ channel optical alignment}

As for the HRI $\mathrm{HUV}_{\mathrm{EUV}}$ channel, the two mirrors of the HRI Lyman-alpha (HRI $\mathrm{Hya}_{\mathrm{L}}$ ) channel have been placed using theodolites and 3D arm to check their orientation w.r.t. the reference cube (UARF) and using a 3D arm to check their position w.r.t. theoretical position from the optical model.

Prior to mirror final installation on the flight optical bench, the interferometric alignment of the $\mathrm{HRI}_{\mathrm{Lya}}$ mirrors has been performed on a dummy bench, and then been transferred to the flight bench by geometrical verification of the alignment (orientations and positions of the mirrors using the 3D arm and theodolites). However, interferometric verification on the flight bench proved unfeasible due to lack of room and accessibility, inside the central part of the OBS housing. The verification of focus position was deemed unnecessary due to the large tolerance of this optical design. Figure 10 shows the aligned $\mathrm{HRI}_{\mathrm{Lya}}$ channel with its camera installed.

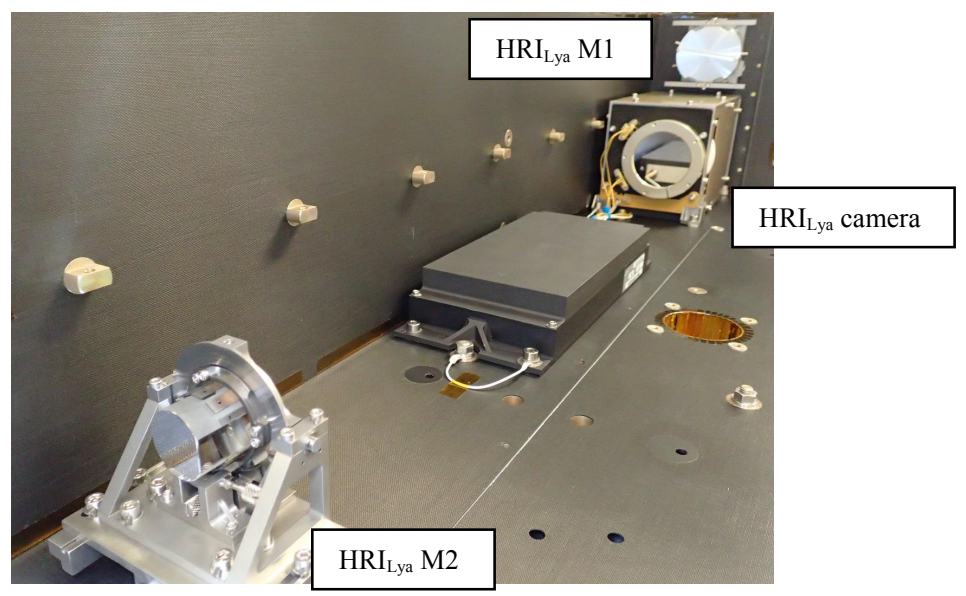

Figure 10 - EUI HRI $\mathrm{Lya}_{\mathrm{a}}$ channel primary and secondary mirror set in place within OBS cavity.

\subsection{FSI channel optical alignment}

The FSI channel was simpler, as only one mirror has to be aligned. The main steps were the mirror alignment with regards to the mechanical axis, alignment of a dummy camera on axis and for different positions in the FOV using an interferometer, and then integration of the flight camera.

3D measurements of the FSI channel were performed to assess the correct positioning of the mirror and the camera, and the FSI channel filter wheel was also aligned within the tolerance budget of $\pm 0.2^{\circ}$ w.r.t. the optical axis.

A fine tuning of the alignment of the camera was been performed using a dummy camera consisting of an array of micro lenses centred on each FOV. Due to the lack of time, the alignment has however only been performed using the central FOV and verified for three other FOVs. At the end of the process, the measured distance between the focal plane and the mirror is only $20 \mu \mathrm{m}$ from the theoretical value, the FSI channel being so aligned within specified tolerances (Figure 11).

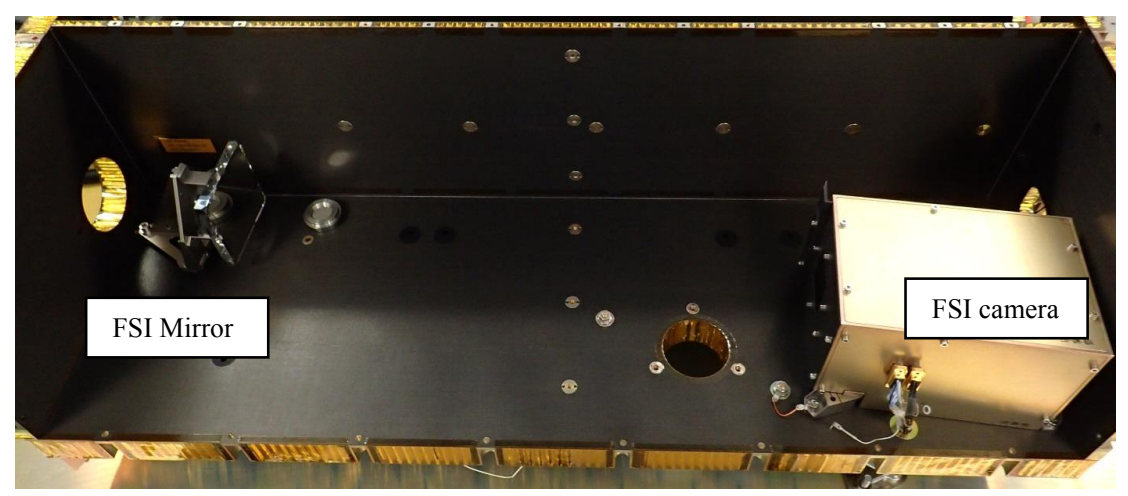

Figure 11 - EUI FSI channel mirror and camera set in place within OBS cavity. 


\subsection{Channel co-alignment}

Each channel was thus interferometrically aligned to ensure best performance but also co-aligned as good as possible with respect to the reference cube. The Instrument Line of sight (ILS) was then considered as the average of the two HRI channels LoS (the channels with smaller field of views).

The ILS offset vs. UARF (front reference cube) and vs. URF (mechanical axis) is summarised in Table 1, where the offset accuracy is $<20$ arcsec.

Table 1 - EUI ILS w.r.t. UARF and URF reference frames.

\begin{tabular}{|c|c|c|c|c|}
\hline & ILS vs. UARF & UARF vs. URF & ILS vs. URF & \\
\hline $\mathbf{H z}$ & $-1 \operatorname{arcsec}$ & $+70 \operatorname{arcsec}$ & $+69 \operatorname{arcsec}$ & + clockwise (around + ZEUI) - YawEUI \\
\hline $\mathbf{V}$ & $+7 \operatorname{arcsec}$ & $-60 \operatorname{arcsec}$ & $-53 \operatorname{arcsec}$ & + clockwise (around + YEUI) - PitchEUI \\
\hline
\end{tabular}

\section{END-TO-END CALIBRATION TEST}

The aim of the end-to-end calibration was to provide adequate characterisation of EUI flight model instrumental response for its scientific use. The general principle is to compare the EUV signal measured by the EUI telescope with the signal measured by a calibrated reference standard, to have an absolute end-to-end radiometric calibration of the whole EUI optical system that is composed of the bandpass filters, the mirrors reflective coatings, and the detectors.

\subsection{Test setup}

The EUI flight instrument was calibrated with synchrotron radiation from the Metrology Light Source (MLS) of the Physikalisch-Technische Bundesanstalt (PTB) in Berlin ${ }^{[14]}$. The OBS unit was mounted inside a dedicated high-vacuum tank on a support bracket fixed on a translation/rotation stage, allowing to scan the channel's FOV. The tank was connected to the MLS undulator (IDB). Figure 12 and Figure 13 show the vacuum chamber at PTB facility and the calibration test setup.
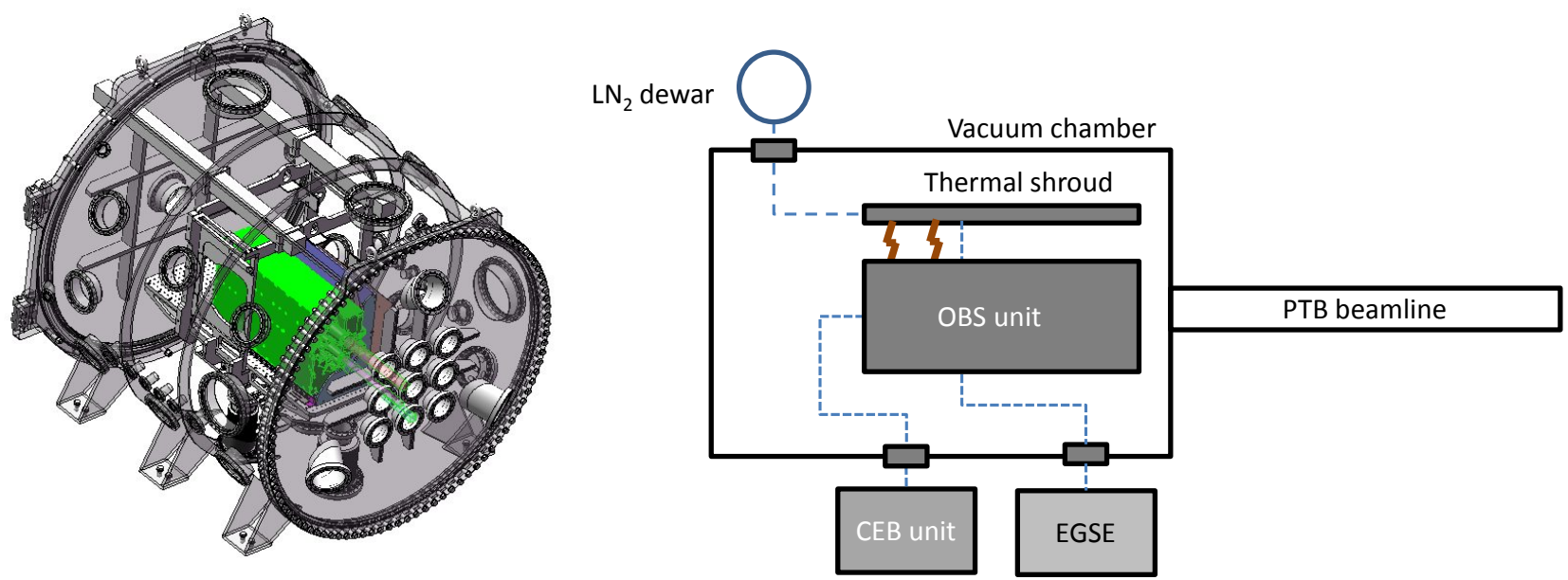

Figure 12 - Left: vacuum chamber at PTB facility with OBS unit located inside. Right: schematic overview of the calibration test setup at the PTB facility.

The EUV beam at the MLS IDB has a small divergence $(<2 \mathrm{mrad})$ and was therefore not intended to be used to check the alignment and focus but rather to concentrate on radiometric and spectral performances of the instrument channels in their respective operational wavelengths and over their FOV. A straylight verification (incident EUV light source out of the channel field of views) was also planned as part of this calibration.

To ensure reliable measurements with limited thermal noise, the EUI detectors were cooled down while the rest of the OBS unit remained at room temperature. The CEB unit, used to operate the cameras and control the OBS mechanisms 
(filter wheels and door), was located out of the chamber, and electrically connected to the OBS through electrical feedthroughs (Figure 13).
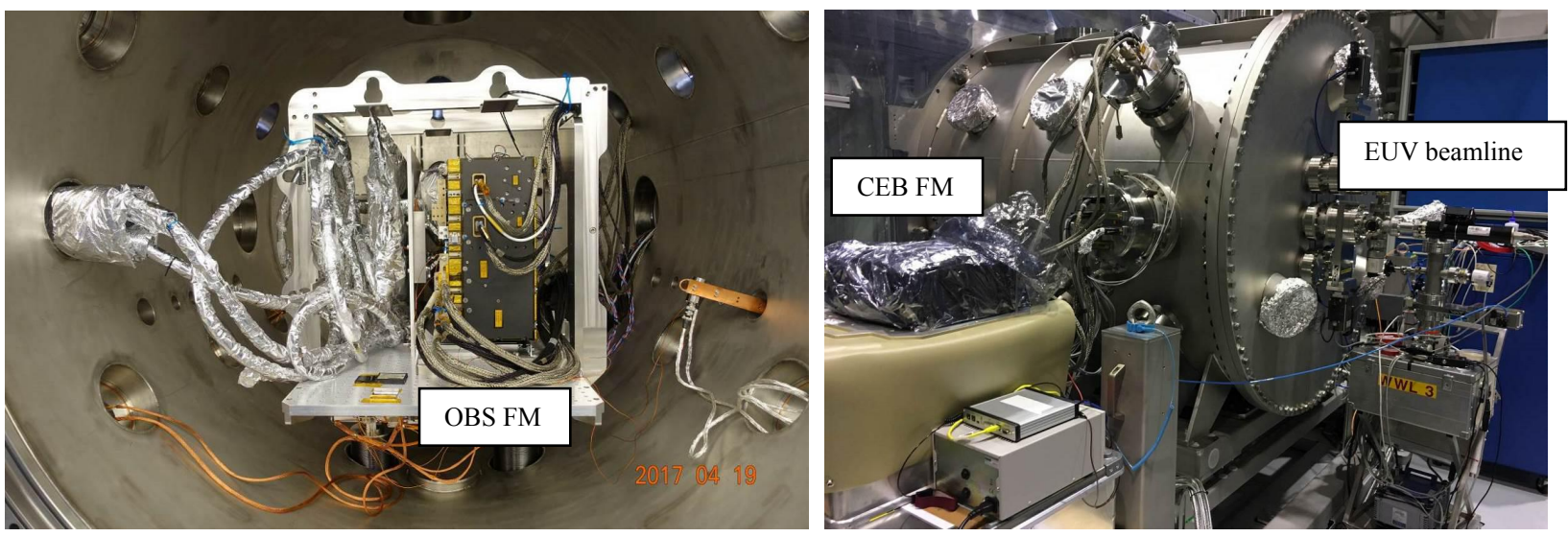

Figure 13 - Left: OBS flight unit located in the PTB vacuum chamber. Right: CEB located outside and vacuum chamber connection to the MLS beam line.

\subsection{Calibration results}

Detailed analysis of the entire calibration takes time and only preliminary results can be reported here. They show already the good behaviour of the three channels and their response to the EUV beam input was as expected.

Figure 14 shows the relative spectral response of the FSI channel from $15 \mathrm{~nm}$ to $34 \mathrm{~nm}$, and a combined image of the beam spot at $17.4 \mathrm{~nm}$ in the corners and centre of the FSI channel FOV ( $3072 \times 3072$ detector pixels).
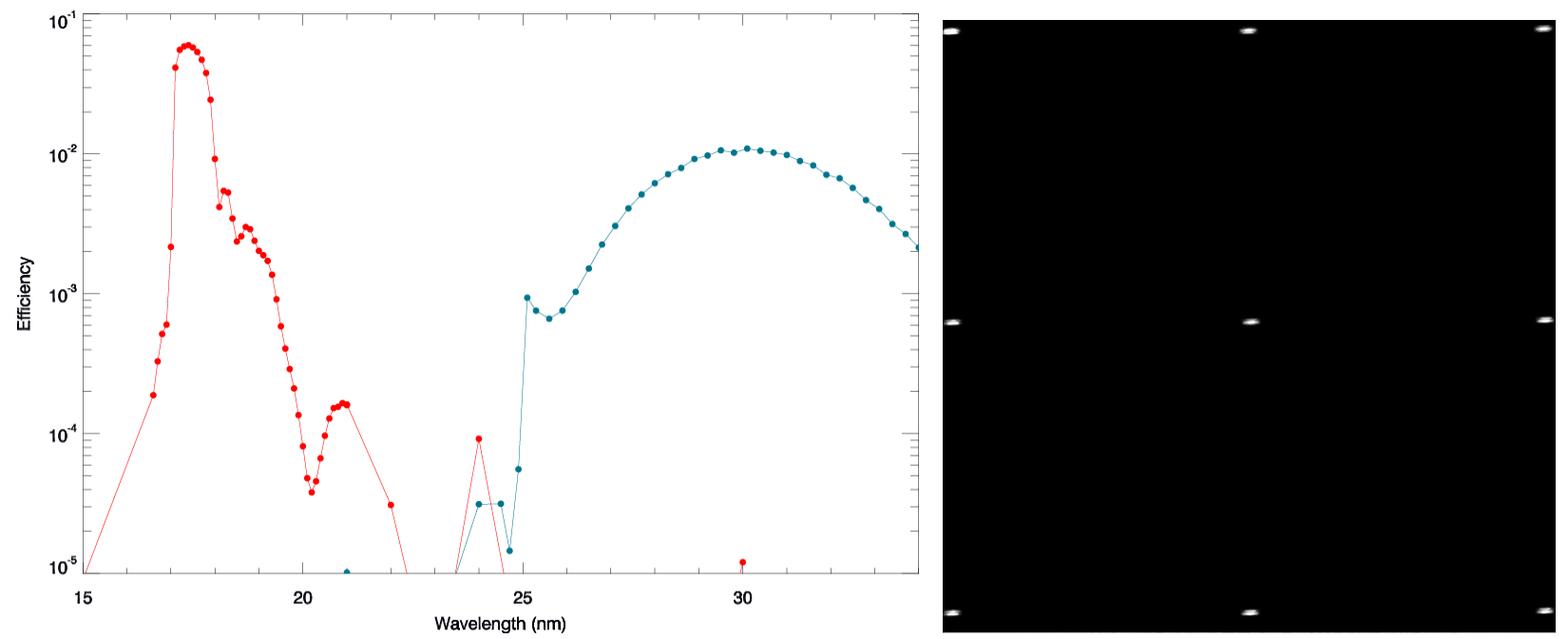

Figure 14 - Left: relative spectral response of the FSI channel from $15 \mathrm{~nm}$ to $34 \mathrm{~nm}$. Right: combined image with the $17.4 \mathrm{~nm}$ beam spot in the corners and centre of the FSI channel FOV.

In Figure 15 we compare the spectral response of the FM units of the entire Ly-alpha channel with the response of the Ly- $\alpha$ camera.

The ratio of the two measurements represents the efficiency of the two mirror coatings and the two Lyman-Alpha filters inside the telescope. The upper curve shows the spectral response of the Ly-alpha camera (asterisks), obtained in the spectral range from $115 \mathrm{~nm}$ to $310 \mathrm{~nm}$, and the lower curve represents the spectral response of the Ly-alpha telescope (diamonds) in the range from $115 \mathrm{~nm}$ to $139 \mathrm{~nm}$. Low and high gain channel measurements of the camera are represented with different colours. The orange solid and dashed lines represent the theoretical response of the Ly-alpha camera and the whole Ly-alpha instrument, respectively, as previously determined by literature values of the subsystems. The black solid line coincides with the instrument response. It has been obtained by shifting $2.6 \mathrm{~nm}$ the theoretical 
response curve, to account for the narrow-band focal plane filter which has a peak transmittance at $119 \mathrm{~nm}$ (while the theoretical response was calculated at $121.7 \mathrm{~nm}$ ). In order to compare the two, the response of the camera, obtained at MCP voltage of $400 \mathrm{~V}$ of the intensifier, had to be re-scaled to a voltage of $545 \mathrm{~V}$ using a scaling factor previously determined by a calibration of the intensifier gain-to-voltage ratio. Note, for in-flight observations, the absolute sensitivity (in DN/photon) of the Ly-a Channel can be increased by four orders of magnitude by raising the MCP voltage from $400 \mathrm{~V}$ up to $1000 \mathrm{~V}$.

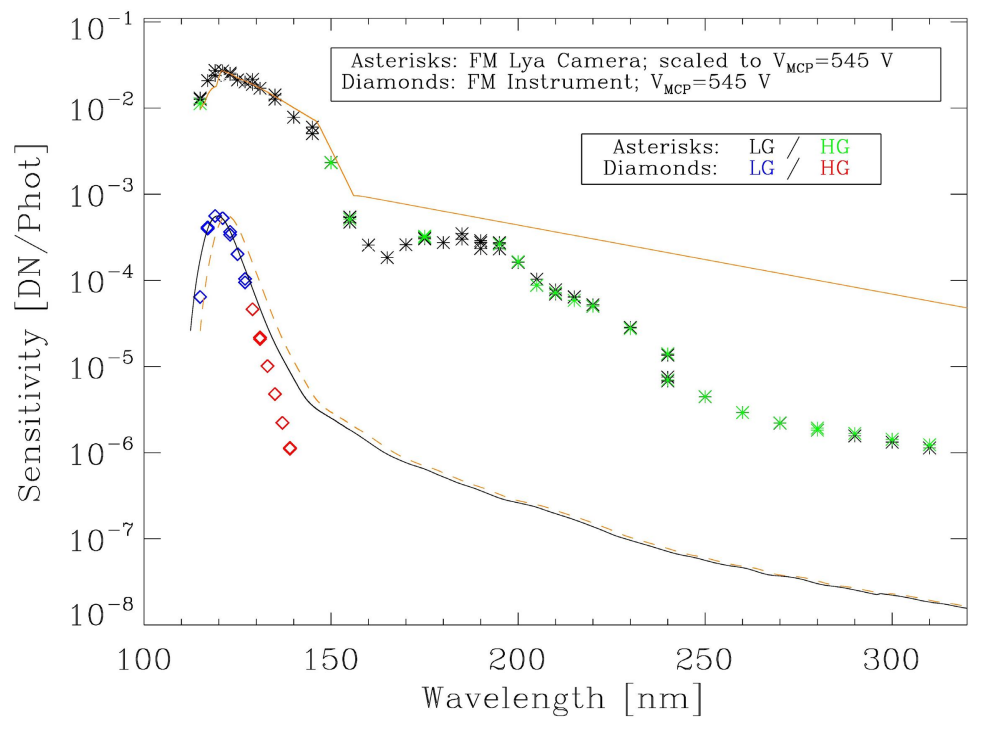

Figure 15 - Spectral sensitivity of the Ly-alpha channel (diamonds) and the previously measured response of the Lyalpha camera (asterisks). Solid lines represent the relative response estimated theoretically during the EUI design phase.

The measurement of the absolute spectral sensitivity of the HRI $\mathrm{EUV}_{\mathrm{V}}$ channel, shown on Figure 16 (left), in the 16-18.5 nm wavelength range was achieved using the high gain channel of the detector in its default settings ant at a detector temperature of $-35^{\circ} \mathrm{C}$. The sensitivity peak is estimated to be at $17.4 \mathrm{~nm}$. The PTB-MLS EUV beam flux was lowered as much as possible to protect the detector against possible degradation by EUV light. Further analysis to be reporter later, is being performed to assess the absolute efficiency and other key performance of the HRI-EUV telescope. Figure 16 (right) shows a combined image with the beam spot on the $\mathrm{HRI}_{\mathrm{EUV}}$ detector at the four extreme orientations of the FOV. The red square is the nominal $2048 \times 2048$ pixel readout window, while the blue square shows how far this FOV can be extended). The spot size of the EUV beam is enlarged compared to FSI, due to the higher HRI magnification.
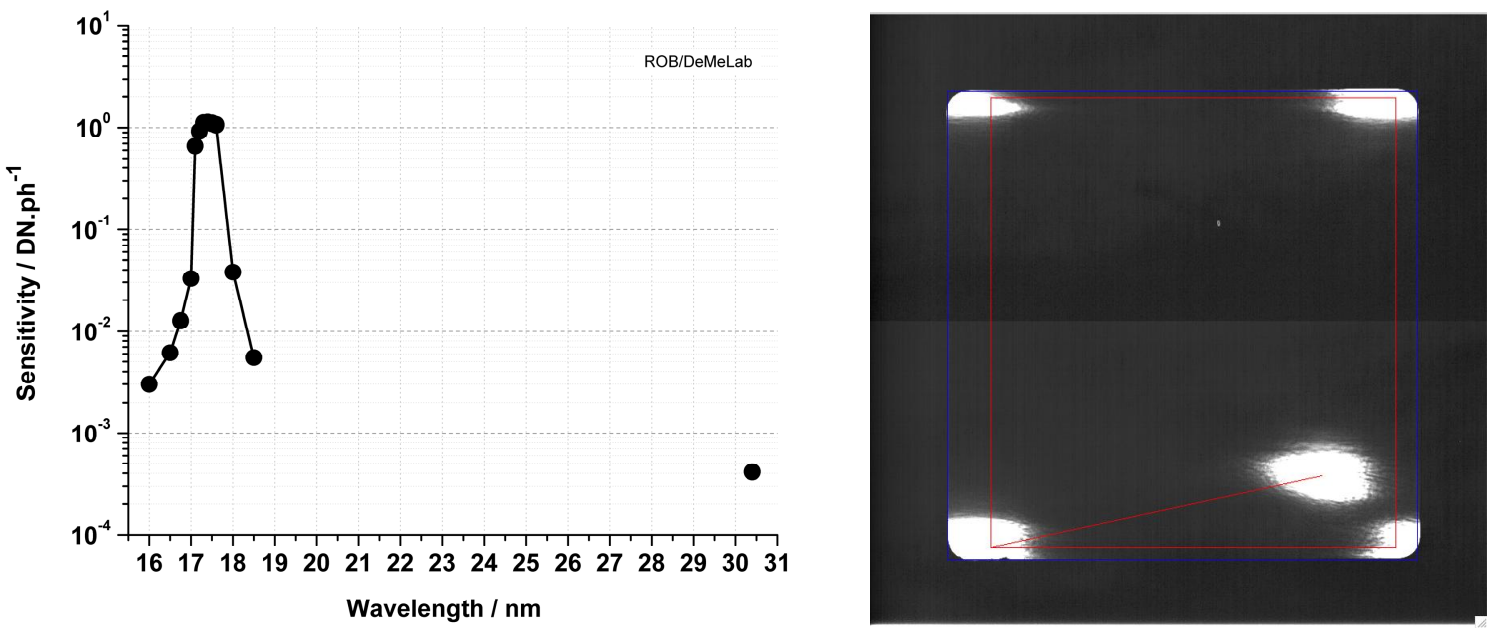

Figure 16 - Left: Spectral sensitivity of the $\mathrm{HRI}_{\mathrm{EUV}}$ channel over the $16-18.5 \mathrm{~nm}$ range and at $30.4 \mathrm{~nm}$. Right: combined image with $17.4 \mathrm{~nm}$ beam spot on the $\mathrm{HRI}_{\mathrm{EUV}} 2048 \times 2048$ detector at the four extreme orientation of the FOV. 


\section{CONCLUSIONS}

The EUI instrument alignment combined with the instrument sub-systems efficiencies (cameras, mechanisms, and CEB), allowed us to achieve the expected performance. The verification of the spectral performances, carried out during the end-to-end calibration, allowed us to validate the complete acquisition chain (mirrors, filters, and detectors to CEB data processing).

The EUI instrument is now installed on-board the Solar Orbiter spacecraft, co-aligned with the other remote-sensing instruments (SPICE, PHI, Metis, STIX), in preparation for the satellite environmental tests and preparation for launch planned in 2020.

The analysis of the calibration data is still on going and will help the in-flight calibration, on-ground processing and interpretation of the flight data, which will be acquired once EUI is operating in space.

\section{ACKNOWLEDGEMENTS}

The EUI instrument was developed in a collaboration including the Centre Spatial de Liège (Belgium), the Institut d'Astrophysique Spatiale and the Institut d'Optique (France), the UCL Mullard Space Science Laboratory (UK), the Max Planck Institute for Solar System Research (Germany), the Physikalisch-Meteorologisches Observatorium Davos (Switzerland), and the Royal Observatory of Belgium (Belgium).

The Belgian institutes are funded by Belgian Federal Science Policy Office (BELPSO); the French institutes by Centre National d'Etudes Spatiales (CNES); the UK institute by the UK Space Agency (UKSA); the German institute by Deutsche Zentrum für Luft- und Raumfahrt e.V. (DLR), and the Swiss institute by the Swiss Space Office (SSO).

\section{REFERENCES}

[1] Rochus P., Halain J.P., Renotte E., Berghmans D., Zhukov A., Hochedez J.F., Appourchaux T., Auchère F., Harra L.K, Schühle U., Mercier R.., "The Extreme Ultraviolet Imager (EUI) on-board the Solar Orbiter Mission" 60th International Astronautical Congress, (2009).

[2] Hochedez J.-F., Appourchaux T., Defise J.-M., Harra L. K., Schuehle U., Auchère F., Curdt W., Hancock B., Kretzschmar M., Lawrence G., Marsch E., Parenti S., Podladchikova E., Rochus P., Rodriguez L., Rouesnel F., Solanki S., Teriaca L., Van Driel L., Vial J.-C., Winter B., Zhukov A., "EUI, The Ultraviolet Imaging Telescopes of Solar Orbiter" The Second Solar Orbiter Workshop, (2006).

[3] Halain J.-P., Rochus P., Appourchaux T., Berghmans D., Harra L., Schühle U., Auchère F., Zhukov A., Renotte E., Defise J.-M., Rossi L., Fleury-Frenette K., Jacques L., Hochedez J.-F., Ben Moussa A., "The technical challenges of the Solar-Orbiter EUI instrument" Proc. SPIE 7732, 26 (2010)

[4] Schühle U., Halain J., Meining S., Teriaca L., "The Lyman-alpha telescope of the extreme ultraviolet imager on Solar Orbiter" Proc. SPIE Solar Physics and Space Weather Instrumentation IV, 8148, (2011)

[5] Halain J.-P., Rochus P., Renotte E., Appourchaux T., Berghmans D., Harra L., Schühle U., Schmutz W., Auchère, F., Zhukov A., Dumesnil C., Kennedy, T., Mercier R., Pfiffner D., Rossi L., Tandy J., Smith P., "The EUI instrument on board the Solar Orbiter mission: from breadboard and prototypes to instrument model validation" Proc. SPIE 8443, (2012)

[6] Halain JP, Debaize A., Gillis JM., Jacques L., De Ridder T., Hermans L., Koch M., Meynant G., Schippers G., "The dual-gain $10 \mu \mathrm{m}$ back-thinned 3k x 3k CMOS-APS detector of the Solar Orbiter Extreme UV Imager" Proc. SPIE, 9144, (2014)

[7] Halain JP, Rochus P, Renotte E, Auchère F, Berghmans D, Harra L, Schühle U, Schmutz W, Zhukov A, Aznar Cuadrado R, Delmotte F, Dumesnil C, Gyo M, Kennedy T, Mercier R, Verbeeck C, Thome M, Heerlein K, Hermans A, Jacques L, Mazzoli A, Meining S, Rossi L, Tandy J, Smith P, Winter B, "The Extreme UV Imager of Solar Orbiter - From detailed design to Flight Model" Proc SPIE, 9144, (2014)

[8] Rossi L, Zhukova M, Jacques L, Halain JP, Hellin ML, Jamotton P, Renotte P, Rochus P, Liebecq S, Mazzoli A, "Solar simulation test up to 13 solar constants for the thermal balance of the Solar Orbiter EUI instrument" Proc SPIE, 9144, (2014) 
[9] Halain JP, Rochus P, Renotte E, Hermans A, Jacques L, Auchère F, Berghmans D, Harra L, Schühle U, Schmutz W, Zhukov A, Aznar Cuadrado R, Delmotte F, Dumesnil C, Gyo M, Kennedy T, Smith P, Tandy J, Mercier R, Verbeeck C, "The Extreme UV Imager telescope on-board the Solar Orbiter mission - Overview of phase C and D" Proc SPIE, 9604, (2015)

[10] Halain JP, Mazzoli A, Meining S, Rochus P, Renotte E, Auchère F, Schühle U, Delmotte F, Dusmenil C, Philippon A, Mercier R, Hermans A, "The Extreme Ultraviolet Imager of Solar Orbiter: Optical Design and Alignment Scheme" Proc SPIE, 9604, (2015)

[11] Thomas Reichel et al., "Developments in calibration of EUV and VUV detectors for Solar Orbiter instrumentation using synchrotron radiation" Proc SPIE, 9905-120, (2016)

[12] Roman M. Klein et al., Source-based calibration of space instruments using synchrotron radiation at the metrology light source Proc SPIE, 9905-122, (2016)

[13] Halain JP, Rochus P, Renotte E, Hermans A, Jacques L, Mazzoli A, Auchère F, Berghmans D, Harra L, Schühle U, Schmutz W, Aznar Cuadrado R, Dumesnil C, Gyo M, Kennedy T, Verbeeck C, Smith P, "The qualification campaign of the EUI instrument of Solar Orbiter" Proc SPIE, 9905-141, (2016)

[14] R. Klein, R. Fliegauf, A. Gottwald, M. Kolbe, W. Paustian, T. Reichel, M. Richter, R. Thornagel, and G. Ulm, "Calibration of space instruments at the Metrology Light Source", AIP Conference Proceedings 1741, 030013 (2016) 